\title{
Effect of Annealing on Magnetostrictive Properties of Fe-Co Alloy Thin Films
}

\author{
Takashi Nakajima ${ }^{1, *}$, Teruaki Takeuchi ${ }^{2}$, Isamu Yuito ${ }^{2}$, Kunio Kato $^{2}$, Mikiko Saito ${ }^{2}$, \\ Katsuhiro Abe ${ }^{1}$, Toshio Sasaki ${ }^{1}$, Tetsushi Sekiguchi ${ }^{2}$ and Shin-ichi Yamaura ${ }^{1}$ \\ ${ }^{1}$ Institute for Materials Research, Tohoku University, Sendai 980-8577, Japan \\ ${ }^{2}$ Institute for Nanoscience \& Nanotechnology, Waseda University, Tokyo 162-0041, Japan
}

\begin{abstract}
The effect of the annealing temperature on the magnetostrictive properties of Fe-Co alloy thin films on quartz glass was systematically investigated. The saturation magnetostriction was $56 \mathrm{ppm}$ for the $\mathrm{Fe}_{32} \mathrm{Co}_{68}$-sputtered thin film quenched from $673 \mathrm{~K}$, and it increased to 159 ppm with increasing annealing temperature up to $1073 \mathrm{~K}$. The magnetostriction above $1093 \mathrm{~K}$ significantly decreased with the emerging fcc phase in the Fe-Co alloy. SEM images showed that the crystal grains present in the fcc phase aggregated to form a discontinuous surface, which resulted in a decrease in the effective magnetostriction. Measurements of the magnetic properties by vibrating sample magnetometer and magnetic force microscopy revealed the enhancement of the magnetization at the crystal grain boundaries of Fe-Co alloy thin films with large magnetostriction. Thus, the heterogeneity of the magnetization can play a key role in inducing the large magnetostrictive effect. [doi:10.2320/matertrans.MBW201315]
\end{abstract}

(Received October 16, 2013; Accepted November 11, 2013; Published December 28, 2013)

Keywords: iron cobalt, thin films, sputtering film deposition, magnetostriction, magnetization, magnetic force microscopy

\section{Introduction}

In recent years, there has been strong interest in introducing magnetostrictive thin films for microelectromechanical systems (MEMS) as novel actuators, sensors, and vibration energy harvesting devices. ${ }^{1-5)}$ A key contribution of MEMS technology is the integration of multifunctional elements on a single chip, which enhances the performance, reliability, and competitive pricing. Rare-earth-based magnetostrictive materials, represented by $\mathrm{Tb}-\mathrm{Dy}-\mathrm{Fe}$, have played a central role in developing such applications owing to their large magnetostriction of more than $2000 \mathrm{ppm}^{.6-11)}$ However, there has also been demand for developing rare-earth-free magnetostrictive materials as a result of the depletion of natural resources.

Recently, the strong magnetostrictive effect originating from the heterogeneity at the phase boundaries of Fe-based alloys has attracted great research interest. Extraordinary magnetostrictive behaviors have been observed in $\mathrm{Fe}-\mathrm{Ga}$ and $\mathrm{Fe}-\mathrm{Al}$ alloys, exhibiting large magnetostriction values $(3 / 2)$ $\lambda_{100}$ of more than $400 \mathrm{ppm}$ for $\mathrm{Fe}_{81.3} \mathrm{Ga}_{18.7^{12,13)}}$ and $200 \mathrm{ppm}$ for $\mathrm{Fe}_{83.4} \mathrm{Al}_{16.6}{ }^{14)}$ In each case, the increase of magnetostriction occurred in the vicinity of ordered $\mathrm{DO}_{3} /$ disordered A2 phase boundaries, and several mechanisms such as magnetic domain rotation ${ }^{15)}$ and large elastic deformation of $\mathrm{DO}_{3}$ nanoclusters ${ }^{16)}$ have been proposed.

Much effort has also been devoted to developing the magnetostriction of $\mathrm{Fe}-\mathrm{Co}$ alloys ${ }^{17-19)}$ ever since the first report on the magnetostrictive properties over the entire composition range by Masiyama. ${ }^{20}$ More recently, Hunter et al. prepared compositional gradient $\mathrm{Fe}-\mathrm{Co}$ alloy thin films using a co-sputtering technique, and they found significant magnetostriction enhancement at the $(\mathrm{fcc}+\mathrm{bcc}) / \mathrm{bcc}$ phase boundary. ${ }^{21)}$ The effective magnetostriction was $260 \mathrm{ppm}$ for an $\mathrm{Fe}_{34} \mathrm{Co}_{66}$ thin film quenched from $1073 \mathrm{~K}$, and the intrinsic magnetostriction $\lambda_{100}$ was estimated to be more

*Corresponding author, E-mail: tnakajima@imr.tohoku.ac.jp than $1000 \mathrm{ppm}$. However, it is difficult to understand the interfacial effect on magnetostriction by focusing solely on its composition dependence because the contribution of Joule magnetostriction, which becomes prominent in the vicinity of $\mathrm{Fe}_{50} \mathrm{Co}_{50}$, ${ }^{21)}$ should also be included. Furthermore, from a practical standpoint in terms of the manufacturing process, it is of special importance to investigate the effect of annealing on the magnetostrictive properties of Fe-Co alloys.

In this study, we report on the annealing temperature dependence of the magnetostriction of $\mathrm{Fe}-\mathrm{Co}$ alloy thin films. Thin films of $\mathrm{Fe}_{32} \mathrm{Co}_{68}$ were deposited on quartz glass using a sputtering technique. The samples were then quenched from 673 to $1173 \mathrm{~K}$. The magnetostrictive properties of these films are discussed in detail based on the characterization of the structures and their magnetic properties.

\section{Experimental Procedure}

Fe-Co alloy thin films $240 \mathrm{~nm}$ thick were sputter-deposited on quartz glass substrates using RFS-200 (ULVAC). The substrates were $20 \mathrm{~mm}$ long, $5 \mathrm{~mm}$ wide, and approximately $60-70 \mu \mathrm{m}$ thick. The films were fabricated at a substrate temperature of $623 \mathrm{~K}$ under an Ar pressure of $1.0 \mathrm{~Pa}$ and a sputtering power of $100 \mathrm{~W}$, which yielded a deposition rate of $2 \mathrm{~nm} / \mathrm{min}$. In this study, the composition of the films was chosen to be $\mathrm{Fe}_{32} \mathrm{Co}_{68}$ so as to obtain an fcc and/or bcc phase by annealing between 673 and $1173 \mathrm{~K}^{22)}$ The samples were annealed for $60 \mathrm{~min}$ in an evacuated glass tube and quenched by immersing the tube in ice water.

The crystallographic phases of the Fe-Co alloy thin films were characterized by X-ray diffraction (XRD; Ultima-III, Rigaku) analysis using $\mathrm{Cu} \mathrm{K} \alpha$ radiation with a monochromator. The microstructure observation and the composition analysis were carried out using a field-emission scanning electron microscope (FE-SEM; S-4800, Hitachi) equipped with an energy dispersive X-ray spectrometer.

The magnetostriction was evaluated by using the cantilever deflection technique, which allows the magnetostriction $\lambda$ 
to be determined from the field-induced bending of the cantilevered substrate. ${ }^{23)}$ The displacement of the cantilever was induced by an alternating magnetic field parallel $\left(H_{\|}\right)$ and perpendicular $\left(H_{\perp}\right)$ to the longitudinal direction of the substrate but always parallel to the film plane. The saturation magnetostriction $\lambda_{\mathrm{s}}$ was calculated using the following expression:

$$
\lambda_{\mathrm{s}}=\frac{\left(\Delta s_{\|}-\Delta s_{\perp}\right) E_{\mathrm{s}} t_{\mathrm{s}}^{2}\left(1+v_{\mathrm{f}}\right)}{18 E_{\mathrm{f}} t_{\mathrm{f}} L l\left(1-v_{\mathrm{s}}\right)}
$$

where $\Delta_{S \|}$ and $\Delta_{S \perp}$ are the maximum position change of the laser spot on the position sensitive detector (PSD) induced by $H_{\|}$and $H_{\perp}$, respectively, $E$ is the Young's modulus, $t$ the thickness, $l$ the distance between the clamping edge to the laser spot on the sample, and $L$ the optical travel distance from the incident point at the sample. The subscripts "s" and "f" denote "substrate" and "film", respectively. The Young's moduli were determined by a nanoindentation technique using a mechanical properties tester for thin films (NEC MH4000). ${ }^{24)}$

The magnetic properties were measured by a vibrating sample magnetometer (VSM; VSM-5-10, TOEI). The magnetic microstructure was investigated by magnetic force microscopy (MFM; SPI3800N/SPA400, SII) employing a silicon cantilever coated with a $\mathrm{CoCr}$ alloy (MFMR-10, NanoWorld).

\section{Results and Discussion}

Figure 1 shows the XRD patterns of the Fe-Co alloy thin films quenched from various temperatures. As shown in Fig. 1(a), the reflections from the bcc and/or the fcc phase were observed in wide-range XRD patterns, depending on the annealing temperature. Detailed data for the crystalline phase of the Fe-Co alloy thin films was furthermore examined by narrow-range XRD patterns and the results are shown in Fig. 1(b). The double-peak feature is due to the $K \alpha_{1}$ and $K \alpha_{2} \mathrm{X}$-rays, but this feature is not detectable at $673 \mathrm{~K}$ owing to the significant broadening of the XRD peak. A single-phase bcc structure was observed when the annealing temperature was less than $1093 \mathrm{~K}$. As the temperature increased, the structure exhibited a coexisting phase of bcc and fcc between 1113 and $1143 \mathrm{~K}$ and single-phase fcc above $1153 \mathrm{~K}$. Thus, the crystal structures, which are consistent with the conventional phase diagram of the $\mathrm{Fe}-\mathrm{Co}$ binary system, ${ }^{22)}$ were successfully obtained.

Figure 2 shows changes in surface morphologies by annealing. The film annealed at $673 \mathrm{~K}$ exhibits a uniform surface that consists of tiny grains. Annealing at $1073 \mathrm{~K}$ resulted in growth of the crystalline grains. The increase of the grain size is supported by the XRD patterns in Fig. 1(b) showing the decrease of the peak width. Annealing at $1133 \mathrm{~K}$ caused the grains to aggregate, thus forming a discontinuous surface. The aggregation was further developed at $1153 \mathrm{~K}$. One of the conceivable causes of the aggregation is the interfacial reaction of the $\mathrm{Fe}-\mathrm{Co}$ layer with the quartz substrate. Bendersky et al. reported the formation of an $\mathrm{Fe}_{2} \mathrm{SiO}_{4}$ mixed silicate at the $\mathrm{FeCo} / \mathrm{SiO}_{2}$ interface by annealing at $1073 \mathrm{~K},{ }^{25)}$ where the silicate may have acted as the crystal-growing nucleus. Differences in the crystal-
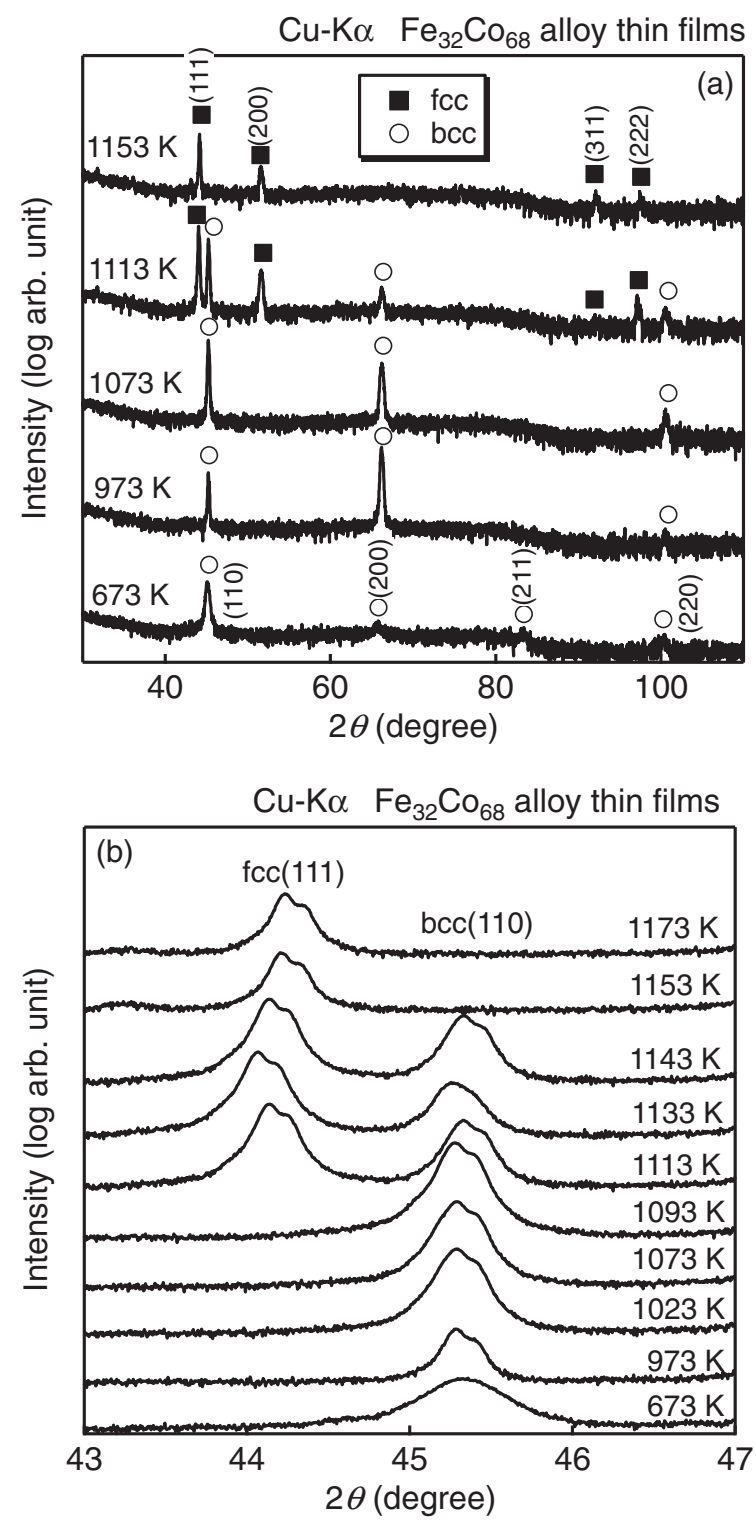

Fig. 1 (a) Annealing temperature dependence of XRD patterns from $2 \theta=30$ to $110^{\circ}$; (b) fcc (111) and bcc (110) reflection peaks for Fe-Co alloy thin films.

lization kinetics between the bcc phase and the fcc phase could be another cause because of the discontinuous surface observed in the film with an fcc phase.

Figure 3 shows the annealing-temperature dependence of $\lambda_{\mathrm{s}}$ of the Fe-Co alloy thin films. The magnetostriction for each sample was determined from the field-induced butterfly loops as a result of magnetostrictive behavior, as shown in the inset of Fig. 3. As the annealing temperature increased, $\lambda_{\mathrm{s}}$ gradually increased, reaching a maximum of $159 \mathrm{ppm}$ at $1073 \mathrm{~K}$ and then it decreased. The $\lambda_{\mathrm{s}}$ value of the sample annealed at $1073 \mathrm{~K}$ is almost three times larger than that of the sample annealed at $673 \mathrm{~K}$. From the XRD results shown in Fig. 1, it is apparent that $\lambda_{\mathrm{s}}$ is greatly enhanced in the vicinity of the bcc/fcc coexisting phase, but note that the phase exhibiting maximum $\lambda_{\mathrm{s}}$ is single bcc. The $\lambda_{\mathrm{s}}$ value decreased with the appearance of the fcc phase, and the magnetostrictive response became undetectable by our measurement system for the fcc single-phase samples annealed above $1143 \mathrm{~K}$. However, the significant decrease 

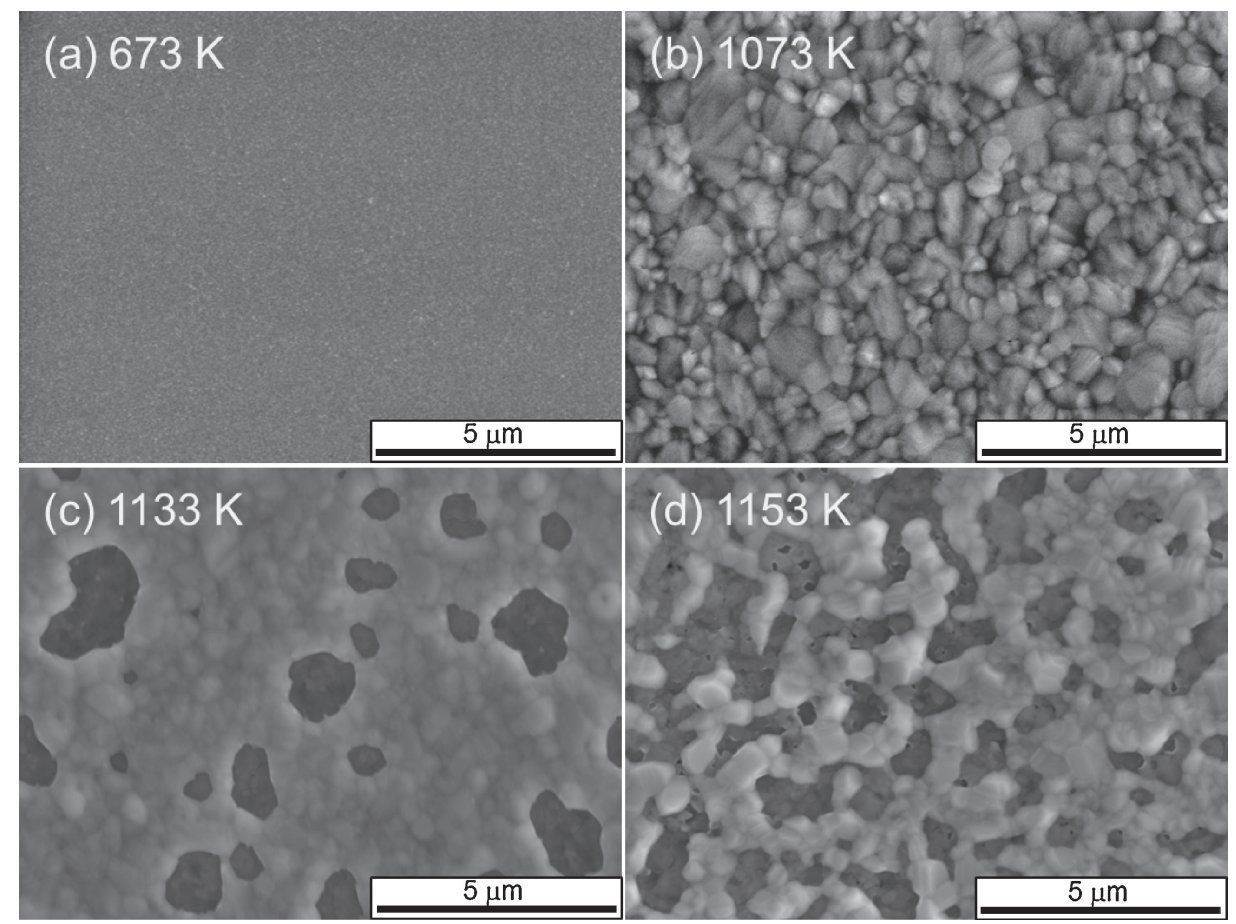

Fig. 2 SEM images of Fe-Co alloy thin films annealed at (a) $673 \mathrm{~K}$, (b) $1073 \mathrm{~K}$, (c) $1133 \mathrm{~K}$, and (d) $1153 \mathrm{~K}$.

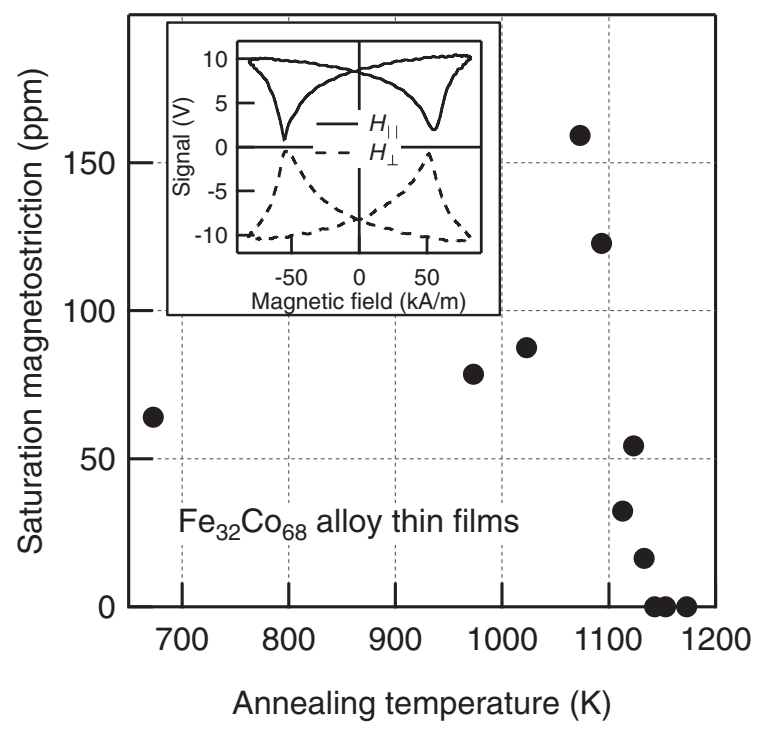

Fig. 3 Annealing temperature dependence of saturation magnetostriction of $\mathrm{Fe}-\mathrm{Co}$ alloy thin films. The inset shows the magnetostrictive behavior of an $\mathrm{Fe}-\mathrm{Co}$ alloy thin film on quartz substrate as detected by the cantilever deflection method.

of $\lambda_{\mathrm{s}}$ is considered to be caused by the discontinuous surface shown in Fig. 2 rather than by the magnetostrictive properties of the crystalline phase. For simplicity in this study, we calculated $\lambda_{\mathrm{s}}$ using eq. (1) on the assumption that the film is uniform, but the intrinsic $\lambda_{\mathrm{s}}$ should be higher because the effective magnetostriction is hindered owing to the discontinuous surface.

Next, we performed $M-H$ curve measurements to evaluate the magnetic properties at room temperature. Figure 4 shows the in-plane hysteresis curves of thin films annealed at 673, 1073 , and $1153 \mathrm{~K}$. Every curve exhibits significant soft

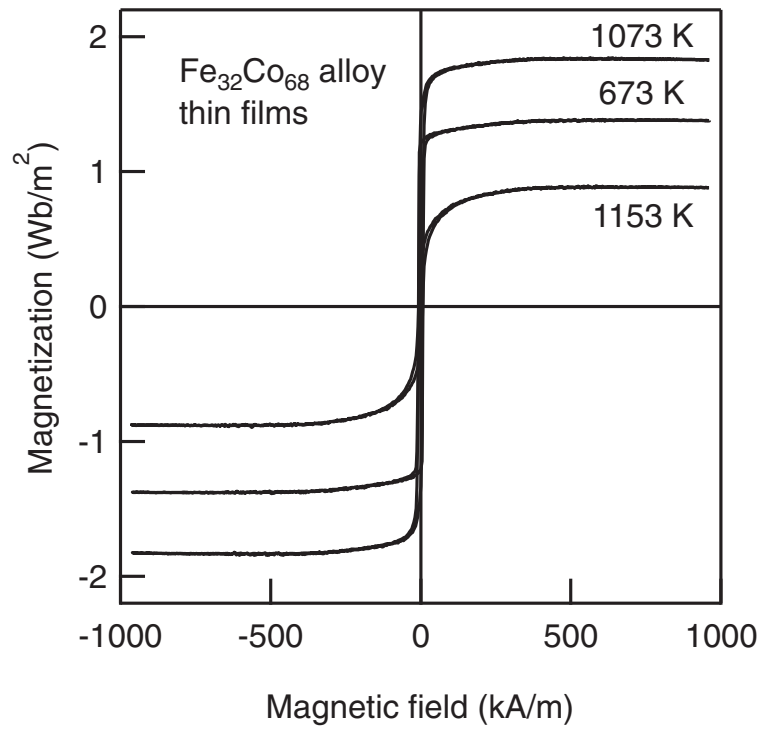

Fig. 4 In-plane magnetization curves of Fe-Co alloy thin films annealed at 673,1073 , and $1153 \mathrm{~K}$.

magnetic properties, indicating that the easy-axis direction of the preferential magnetization is parallel to the film plane. The most striking feature in this figure is the annealing temperature-dependence of the magnetization. Figure 5 shows the plots of saturation magnetization $M_{\mathrm{s}}$ and coercive field $H_{\mathrm{c}}$ against the annealing temperature. It is seen that $M_{\mathrm{s}}$ monotonically increased with increasing annealing temperature up to $1093 \mathrm{~K}$ and then it began to decrease. This tendency is similar to that of the magnetostriction, and the magnetostriction enhancement is considered to be related to the magnetization increase. $H_{\mathrm{c}}$ shows a two-step decrease as the annealing temperature increases. This decrease is probably due to the stress relief created in the material itself 


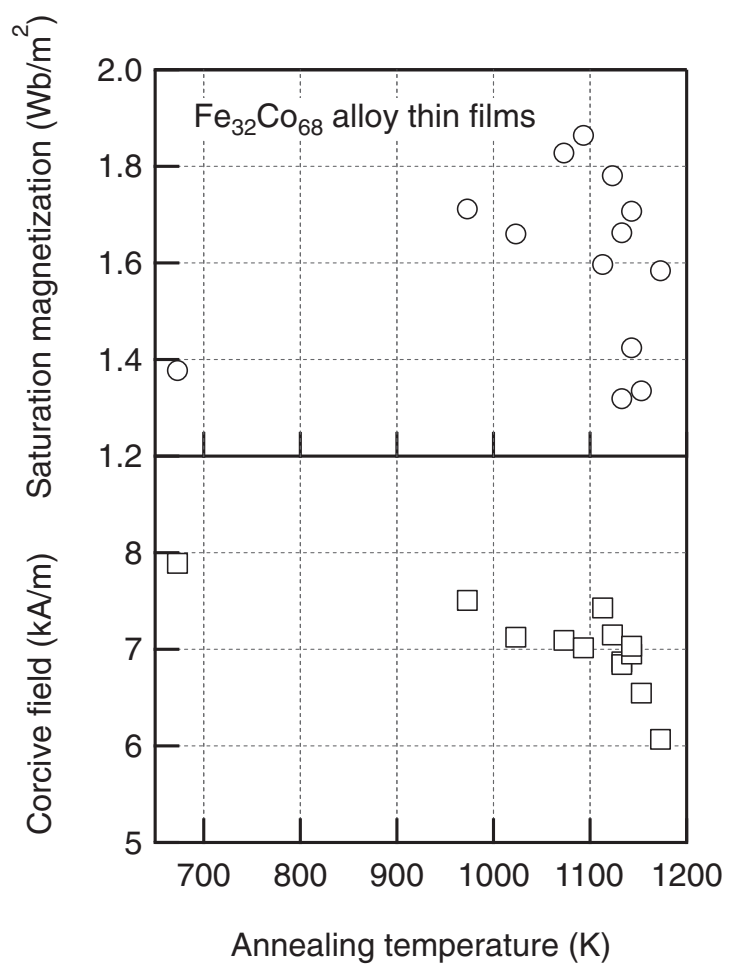

Fig. 5 Annealing temperature dependence of saturation magnetization and coercive field for Fe-Co alloy thin films.

and in the substrate interface, which is estimated to be prominent after the appearance of the discontinuous surface at higher annealing temperatures.

For further understanding of the magnetization increase, we conducted MFM measurements to investigate the magnetic microstructure. Figure 6 shows the results of the MFM measurements on the sample annealed at $1093 \mathrm{~K}$. The AFM image (a) was obtained by tapping mode for the first scan and the MFM image (b) was obtained for the second scan by monitoring the phase shift in the resonant cantilever oscillation, keeping the cantilever height constant above the sample surface configuration. Comparing Figs. 6(a) and 6(b), the MFM image exhibits a dark-colored area in the vicinity of the grain boundaries, as indicated by the arrows. As seen in the line profile of the height and the phase shift shown in Fig. 6(c), the phase shift increases at the dark-colored area. The degree of the phase shift provides information on the magnetic field gradient between the cantilevered tip and the Fe-Co surface. Therefore, these results suggest that the local magnetization is enhanced around the grain interfaces of the $\mathrm{Fe}-\mathrm{Co}$ alloy thin film quenched from $1093 \mathrm{~K}$. The origin of the magnetostriction enhancement in $\mathrm{Fe}-\mathrm{Ga}$ is thought to result from martensitically transformed precipitates in $\mathrm{Fe}-\mathrm{Ga}$ alloys acting as tetragonal defects that can be rotated by applying a magnetic field. ${ }^{15)}$ Hunter et al. also proposed a similar scenario, in which the reorientation of the tetragonal precipitates at the $(\mathrm{fcc}+\mathrm{bcc}) / \mathrm{bcc}$ phase boundary results in the magnetostriction enhancement in $\mathrm{Fe}-\mathrm{Co}$ alloy thin films. ${ }^{21)}$ Our findings of the magnetization increase at the grain boundaries serve as strong evidence for the presence of tetragonal precipitates, which supports the magnetic domain rotation model as the mechanism for the magnetostriction enhancement of the Fe-Co alloy system.
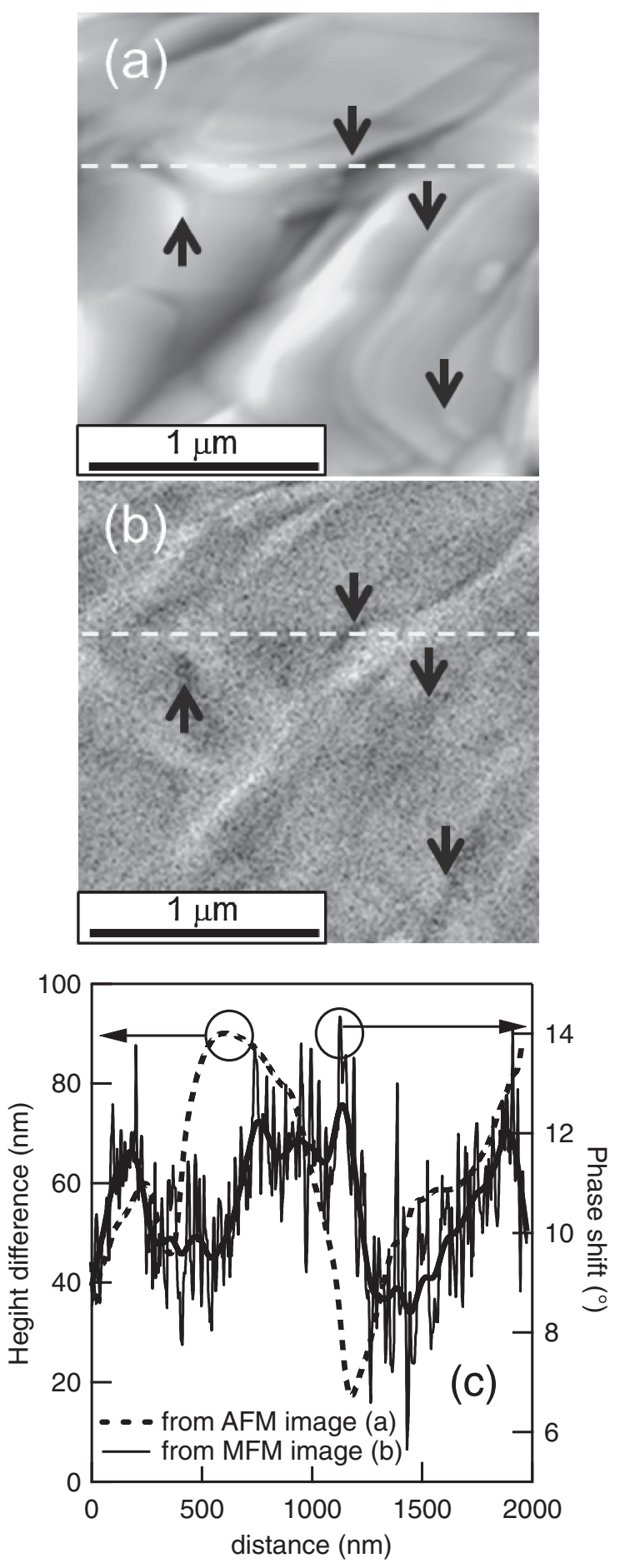

Fig. 6 (a) AFM and (b) MFM images of Fe-Co alloy thin film annealed at 1093 K. (c) Profiles of height and phase shift upon dashed lines in (a) and (b).

\section{Conclusions}

We investigated the effect of annealing temperature on the magnetostrictive properties of a $\mathrm{Fe}_{32} \mathrm{Co}_{68}$ alloy thin film on quartz glass. As the annealing temperature increased, the saturation magnetostriction $\lambda_{\mathrm{s}}$ increased, reaching a maximum of $159 \mathrm{ppm}$ at $1073 \mathrm{~K}$, which is almost three times larger than $\lambda_{\mathrm{s}}$ of the sample annealed at $673 \mathrm{~K}$. We also observed the increase of the magnetization at the grain boundaries of $\mathrm{Fe}-\mathrm{Co}$ alloy thin films with large magneto- 
striction, which would support the magnetic domain rotation model as the mechanism of the magnetostriction enhancement at the $(\mathrm{fcc}+\mathrm{bcc}) / \mathrm{fcc}$ interface. After emerging fccphase annealing above $1113 \mathrm{~K}, \lambda_{\mathrm{s}}$ significantly decreased. SEM observations suggested that the decrease of $\lambda_{\mathrm{s}}$ is due to the discontinuous surface structure rather than to the magnetostrictive properties of the crystalline phase. These findings allow us to envision an ideal structure with large magnetostrictive properties that consist of high density $(\mathrm{fcc}+\mathrm{bcc}) / \mathrm{bcc}$ phase boundaries with suppressed fcc emergence to minimize the undesired discontinuous structure.

\section{Acknowledgments}

This research was supported by a Grant-in-Aid for Science Research in a Priority Area, "Advanced Materials Development and Integration of Novel Structured Metallic Glasses and Inorganic Materials" from the Ministry of Education, Culture, Sports, Science and Technology, Japan. The authors thank Professor T. Furukawa of Kobayashi Institute of Physical Research and Mr. H. Sagami of Ideal Star Inc. for SPM measurements. We are also grateful to Professors R. Y. Umetsu and Y. Mitsui of Tohoku University for valuable discussions.

\section{REFERENCES}

1) D. J. Bell, T. J. Lu, N. A. Fleck and S. M. Spearing: J. Micromech. Microeng. 15 (2005) S153-S164.

2) A. Ludwig and E. Quandt: J. Appl. Phys. 87 (2000) 4691-4695.

3) M. R. J. Gibbs, E. W. Hill and P. J. Wright: J. Phys. D 37 (2004) R237$\mathrm{R} 244$.

4) D. Niarchos: Sens. Actuators A 106 (2003) 255-262.

5) L. Wang and F. G. Yuan: Smart Mater. Struct. 17 (2008) 045009.
6) A. E. Clark: Ferromagnetic Materials, Vol. 1, ed. by E. P. Wohlfarth, (North-Holland, Amsterdam, 1980) pp. 531-589.

7) N. C. Koon, C. M. Williams and B. N. Das: J. Magn. Magn. Mater. 100 (1991) 173-185.

8) H. Szymczak: J. Magn. Magn. Mater. 200 (1999) 425-438.

9) H. Uchida, Y. Matsumura, H. Uchida and H. Kaneko: J. Magn. Magn. Mater. 239 (2002) 540-545.

10) A. G. Olabi and A. Grunwald: Mater. Des. 29 (2008) 469-483.

11) V. H. Babu, G. Markandeyulu and A. Subrahmanyam: Appl. Phys. Lett. 90 (2007) 252513.

12) A. E. Clark, K. B. Hathaway, M. Wun-Fogle, J. B. Restorff, T. A Lograsso, V. M. Keppens, G. Petculescu and R. A. Taylor: J. Appl. Phys. 93 (2003) 8621-8623.

13) E. M. Summers, T. A. Lograsso and M. Wun-Fogle: J. Mater. Sci. 42 (2007) 9582-9594.

14) A. E. Clark, J. B. Restorff, M. Wun-Fogle, D. Wu and T. A. Lograsso: J. Appl. Phys. 103 (2008) 07B310.

15) J. Boisse, H. Zapolsky and A. G. Khachaturyan: Acta Mater. 59 (2011) 2656.

16) H. Cao, P. M. Gehring, C. P. Devreugd, J. A. Rodriguez-Rivera, J. Li and D. Viehland: Phys. Rev. Lett. 102 (2009) 127201.

17) M. D. Cooke, L.-C. Wang, R. Watts, R. Zuberek, G. Heydon, W. M. Rainforth and G. A. Gehring: J. Phys. D 33 (2000) 1450-1459.

18) R. S. Sundar and S. C. Deevi: Int. Mater. Rev. 50 (2005) 157-192.

19) M. Matsumoto, T. Kubota, M. Yokoyama, T. Okazaki, Y. Furuya, A. Makino and M. Shimada: Mater. Trans. 51 (2010) 1883-1886.

20) Y. Masiyama: Sci. Rep. Tohoku Imp. Univ. 1st Ser. 21 (1932) pp. 394 410.

21) D. Hunter, W. Osborn, K. Wang, N. Kazantseva, J. Hattrick-Simpers, R. Suchoski, R. Takahashi, M. L. Young, A. Mehta, L. A. Bendersky, S. E. Lofland, M. Wuttig and I. Takeuchi: Nat. Commun. 2 (2011) 518.

22) I. Ohnuma, H. Enoki, O. Ikeda, R. Kainuma, H. Ohtani, B. Sundman and K. Ishida: Acta Mater. 50 (2002) 379-393.

23) A. C. Tam and H. Schroeder: IEEE Trans. Magn. 25 (1989) 26292638.

24) Y. Tsukamoto, H. Yamaguchi and M. Yanagisawa: Thin Solid Films 154 (1987) 171-181

25) L. A. Bendersky, N. V. Kazantseva, U. R. Kattner, K. Wang, V. P. Oleshko, D. Hunter and I. Takeuchi: Acta Mater. 61 (2013) 4180. 